\title{
Efeito de Humoativo comercial na produtividade da couve-flor no Estado de Santa Catarina
}

\section{Effects of Humoativo commercial in cauliflower productivity in Santa Catarina State}

\author{
José Alfredo da Fonsecal , Ana Lúcia Hanisch ${ }^{2 *}$
}

Resumo: O Humoativo comercial é um substrato registrado como produto orgânico, proveniente do tratamento anaeróbico dos resíduos de celulose em lagoas de decantação e posterior compostagem aeróbica, com alta concentração em nutrientes. O objetivo deste experimento foi avaliar, a campo, o efeito da aplicação de doses de Humoativo sobre a produtividade e a absorção de metais pesados por plantas de couve-flor. Foi utilizado delineamento em blocos inteiramente casualizados, com três repetições e sete tratamentos que corresponderam às doses de Humoativo de 17, 34, 51, 68, 85 e 102 t.ha ${ }^{-1}$, em base seca. $\mathrm{O}$ uso do produto como fertilizante para a cultura da couve-flor proporcionou aumentos crescentes das produtividades com o aumento das doses aplicadas. Não houve efeito dos tratamentos sobre os teores de macro e micronutrientes analisados nos tecidos foliares da couve-flor.

Palavras-chaves: substrato orgânico, Brassica oleracea var. botrytis, metais pesados, resíduo sólido

\begin{abstract}
Humoativo commercial is a substrate organic derived from the anaerobic treatment of waste cellulose into settling ponds, with high concentrations of nutrients. The objective of this experiment was to evaluate, in field, the effect of doses of Humoativo on productivity and on heavy metal uptake by plants of cauliflower. Design was used randomized blocks with three replications and seven treatments that corresponded to doses of Humoativo of 17, 34, 51, 68, 85 and 102 t.ha $^{-1}$ in dry basis. The use of the product as fertilizer for the cultivation of cauliflower provided increases in the yield of culture with increasing doses applied. There was no effect of treatments on the concentrations of macro and micronutrients analyzed in the leaves of cauliflower.
\end{abstract}

Key words: organic substrate, Brassica oleracea var. botrytis, heavy metals, solid residue

\footnotetext{
*Autor para correspondência

Recebido para publicação em 24/04/2014; aprovado em 15/02/2015

${ }^{1}$ Eng-agr., M.Sc., Pesquisador aposentado da Epagri/Estação Experimental de Canoinhas, E-mail zekafonseca@yahoo.com.br

${ }^{2}$ Eng $^{\mathrm{a}}$-agra ${ }^{\mathrm{a}}$, M.Sc., Epagri/Estação Experimental de Canoinhas, E-mail analucia@epagri.sc.gov.br
} 


\section{INTRODUÇÃO}

A preocupação com o desenvolvimento sustentável tem exigido grandes investimentos em pesquisa e desenvolvimento para a melhor alocação dos resíduos da indústria de celulose. O setor produz grande volume de resíduos que precisam ser adequadamente alocados a fim de não intervir no equilíbrio ambiental. Uma das técnicas principais de tratamento de resíduos sólidos é a sua aplicação no solo (ALMEIDA et al., 2007a).

O lodo da estação de tratamento de efluentes (lodo da ETE) - resíduo sólido proveniente do tratamento anaeróbico dos resíduos das fábricas de celulose em lagoas de decantação - tem se transformado em um produto com possibilidade de uso agrícola e apresenta alta disponibilidade na região do Planalto Norte de Santa Catarina. Nesta região, há uma empresa que capta o lodo de ETE, transfere-o para pilhas de compostagem, onde permanecem por um período de 12 a 16 meses, com revolvimentos mensais. Após esse período, o produto resultante é seco e peneirado em leitos cobertos, gerando um produto orgânico com a denominação comercial de Humoativo, um substrato com concentração de nutrientes, registrado para uso em sistemas orgânicos de produção.

Entretanto, devido sua origem, contém também metais pesados. Segundo Nellessen e Fletcher (1993) os metais pesados, normalmente associados à toxidez e a poluição ambiental, são o Arsênio (As), o Cádmio (Cd), o Chumbo $(\mathrm{Pb})$, o Cobre $(\mathrm{Cu})$, o Cromo $(\mathrm{Cr})$, o Mercúrio $(\mathrm{Hg})$, o Molibdênio (Mo), o Níquel (Ni), o Selênio (Se), o Cobalto (Co) e o Zinco (Zn), que, portanto, necessitam ter averiguadas suas disponibilidades quando no solo, bem como sua incorporação pelos tecidos das plantas cultivadas quando do uso desse material como adubo. Diversos autores vem procurando analisar os efeitos de diferentes resíduos na contaminação com metais pesados no solo (MOREIRA et al; 2010; PINTO FILHO et al., 2012) e em plantas (OLIVEIRA et al, 2002; SILVA et al., 2006).

$\mathrm{Na}$ cultura da couve-flor (Brassica oleracea var. botrytis) a qualidade e a produtividade são influenciadas pelo manejo da adubação, dentre outros fatores. As hortaliças, de um modo geral, caracterizam-se por demandar materiais orgânicos como insumo, tendo em vista serem favorecidas pela disponibilização gradativa dos nutrientes por todo o ciclo das culturas (SOUZA; RESENDE, 2002). O Humoativo comercial oferece, teoricamente, características que atendem essa demanda. Embora $\mathrm{K}$ e $\mathrm{N}$ sejam extraídos em maiores quantidades pela couve-flor, experimentalmente, o $\mathrm{P}$ e o $\mathrm{N}$ são os nutrientes que tem fornecido maiores respostas em produtividade (KANO et al., 2010). Segundo Holzschuh et al. (2003) em condições de baixo teor de P no solo, o rendimento de cabeças pode ser inferior a $20 \%$ do rendimento máximo.

Com este trabalho objetivou-se avaliar o efeito da aplicação de doses de Humoativo comercial sobre a produção e absorção de nutrientes, especialmente de metais pesados, por plantas de couve-flor.

\section{MATERIAL E MÉTODOS}

O experimento foi instalado na Estação Experimental da Epagri de Canoinhas, SC $\left(26^{\circ} 22^{\prime} 15^{\prime}\right.$ 'S e $50^{\circ} 16^{\prime} 37^{\prime \prime} \mathrm{W}$, altitude de $800 \mathrm{~m}$ e clima $\mathrm{Cfb}$ ) em um Latossolo Vermelho Distrófico típico, cujo pH foi elevado a 6,0, através da aplicação de calcário dolomítico seguindo recomendações da CQFS-RS/SC (2004). Na Tabela 1 observa-se as características do solo, por blocos, prévia à instalação do experimento.

Tabela 1 - Indicadores químicos dos solos, por blocos, anteriormente à implantação do experimento. Canoinhas, SC, 2008

\begin{tabular}{lllll}
\hline Determinação & Bloco 1 & Bloco 2 & Bloco 3 & Unidade \\
\hline Textura & 68 & 67 & 69 & $\%$ argila \\
pH $\mathrm{H}_{2} \mathrm{O}$ & 5,10 & 6,20 & 5,00 & --- \\
Fósforo & 10,60 & 7,10 & 6,70 & $\mathrm{mg} \cdot \mathrm{kg}^{-1}$ \\
Potássio & 0,52 & 0,67 & 0,81 & $\mathrm{cmol} \cdot \mathrm{kg}^{-1}$ \\
Cálcio & 5,30 & 9,60 & 6,30 & $\mathrm{cmol}_{\mathrm{c}} \cdot \mathrm{kg}^{-1}$ \\
Magnésio & 2,40 & 5,00 & 1,40 & $\mathrm{cmol}_{\mathrm{c}} \cdot \mathrm{kg}^{-1}$ \\
Sódio & 0,039 & 0,035 & 0,052 & $\mathrm{cmol}_{\mathrm{c}} \cdot \mathrm{kg}^{-1}$ \\
Alumínio & 0,90 & 0,00 & 1,70 & $\mathrm{cmol}_{\mathrm{c}} \cdot \mathrm{kg}^{-1}$ \\
$\mathrm{H}+$ Al & 12,26 & 3,47 & 13,75 & $\mathrm{cmol}_{\mathrm{c}} \cdot \mathrm{kg}^{-1}$ \\
Sat. Bases $-\mathrm{V} \%$ & 40,25 & 81,52 & 38,40 & $\mathrm{cmol} \cdot \mathrm{kg}^{-1}$ \\
CTC & 20,52 & 18,78 & 22,32 & $\mathrm{cmol} \cdot \mathrm{kg}^{-1}$ \\
Mat. Orgânica & 47,00 & 53,00 & 58,00 & $\mathrm{~g} \cdot \mathrm{kg}^{-1}$ \\
Ferro & 0,40 & 0,43 & 0,39 & $\mathrm{~g} \cdot \mathrm{kg}^{-1}$ \\
Manganês & 7,80 & 4,40 & 11,24 & $\mathrm{mg} \cdot \mathrm{kg}^{-1}$ \\
Zinco & 1,28 & 2,68 & 2,36 & $\mathrm{mg} \cdot \mathrm{kg}^{-1}$ \\
Boro & 0,19 & 0,28 & 0,15 & $\mathrm{mg} \cdot \mathrm{kg}^{-1}$ \\
Cobre & 1,36 & 0,76 & 1,40 & $\mathrm{mg} \cdot \mathrm{kg}^{-1}$ \\
\hline
\end{tabular}

Foi utilizado delineamento em blocos inteiramente casualizados, com três repetições e sete tratamentos que corresponderam às doses de Humoativo de 17, 34, 51, 68, 85 e 102 t.ha $^{-1}$, em base seca. As quantidades de Humoativo foram definidas em função do fornecimento de $\mathrm{P}_{2} \mathrm{O}_{5}$ à cultura sendo que a recomendação para a cultura da couve-flor, segundo a CQFS-RS/SC (2004) é 180 kg.ha ${ }^{-1}$, o que correspondeu ao tratamento com $51 \mathrm{t}^{-h^{-1}}$ do produto. Algumas características físicas e químicas do Humoativo comercial encontram-se na Tabela 2. Foi utilizado ainda um tratamento referência, com a aplicação de $180 \mathrm{~kg} \cdot \mathrm{ha}^{-1}$ de $\mathrm{P}_{2} \mathrm{O}_{5}$ na forma de superfosfato triplo (SFT). Cada parcela experimental possuía área total de $20 \mathrm{~m}^{2}(5 \times 4 \mathrm{~m})$ e área útil de $12,2 \mathrm{~m}^{2}$, na área central da parcela.

Tabela 2 - Características químicas e físicas do Humoativo

\begin{tabular}{ccc}
\hline Parâmetros & Unidade & Valor \\
\hline $\mathrm{pH}_{\mathrm{H} 2 \mathrm{O}}$ & --- & 6,50 \\
Umidade a $75^{\circ} \mathrm{C}$ & $\%$ & 56 \\
Carbono orgânico & $\mathrm{g} \cdot \mathrm{kg}^{-1}$ & 440 \\
Densidade & --- & 0,90 \\
$\mathrm{P}_{2} \mathrm{O}_{5}$ & $\mathrm{~g} \cdot \mathrm{kg}^{-1}$ & 8,00 \\
$\mathrm{~K}_{2} \mathrm{O}$ & $\mathrm{g} \cdot \mathrm{kg}^{-1}$ & 3,60 \\
$\mathrm{~N}$ total & $\mathrm{g} \cdot \mathrm{kg}^{-1}$ & 10,00 \\
$\mathrm{Na}$ total & $\mathrm{mg} \cdot \mathrm{kg}^{-1}$ & 578,00 \\
$\mathrm{~S}$ total & $\mathrm{g} \cdot \mathrm{kg}^{-1}$ & 8,30 \\
$\mathrm{CaO}$ & $\mathrm{g} \cdot \mathrm{kg}^{-1}$ & 25,00 \\
$\mathrm{MgO}$ & $\mathrm{g} \cdot \mathrm{kg}^{-1}$ & 4,50 \\
$\mathrm{~B}$ total & $\mathrm{mg} \cdot \mathrm{kg}^{-1}$ & 11,00 \\
$\mathrm{Cu}$ total & $\mathrm{mg} \cdot \mathrm{kg}^{-1}$ & 40,00 \\
$\mathrm{Zn}$ total & $\mathrm{mg} \cdot \mathrm{kg}^{-1}$ & 83,00 \\
\hline
\end{tabular}

Determinações conforme metodologia descrita por Tedesco et al. (1995)

Foi utilizada a cultivar Júlia de couve-flor, recomendada para a época de realização do experimento. As sementes 
foram semeadas em bandejas de poliestireno expandido de 128 células contendo substrato comercial. As mesmas permaneceram em casa de vegetação por 25 dias, quando estavam emitindo a segunda folha definitiva, momento em que as mudas foram transplantadas para os canteiros, com espaçamento de $0,8 \times 0,6 \mathrm{~m}$ entre covas.

O plantio foi realizado em agosto de 2008 , em covas, às quais foram adicionadas $1 \mathrm{~g} /$ cova de bórax. Tendo em vista o alto volume do Humoativo a ser utilizado, metade da quantidade foi aplicada a lanço e incorporado ao solo antes do plantio e a outra metade foi aplicada na cova e misturada ao solo, seguindo-se o plantio. Foram realizadas quatro aplicações de nitrogênio em cobertura, na forma de uréia, nas épocas recomendados (CQFS-RS/SC, 2004), visando complementar as quantidades requeridas pela cultura e não suficientes no Humoativo. Não houve necessidade de controle fitossanitário.

Para realização da análise foliar foram coletadas quatro folhas completamente desenvolvidas por ocasião da formação da cabeça, em cada planta da área útil da parcela, de acordo a metodologia proposta por Jones, Wolf e Mills (1991) e pela recomendação da CQFS RS/SC (2004) para a cultura. Após a coleta, as folhas foram lavadas e colocadas para secar em estufa com circulação forçada de ar a $65^{\circ} \mathrm{C}$, até atingir peso constante. Após a secagem, as folhas foram trituradas em moinho tipo Willey e encaminhadas para análise laboratorial dos teores de N (Kjeldahl 0,01\%), P, K, Ca, Mg, S, Na, Zn, $\mathrm{Cu}, \mathrm{Mn}$ e Fe (TEDESCO et al., 1995) e dos metais pesados $\mathrm{Cd}, \mathrm{Cr}, \mathrm{Pb}$ (USEPA EPA 3050, 1999) e Hg (USEPA 7471, 1999). A produtividade foi determinada pela colheita das inflorescências que foram pesadas na medida em que foram atingindo o ponto de colheita recomendado para obtenção do produto desejado pelo mercado.

Os dados coletados foram submetidos à análise de variância e teste $\mathrm{F}$ com o auxílio do programa SISVAR e, quando constatada diferença entre tratamentos ao nível de 5\% de probabilidade do erro os dados foram submetidos à análise de regressão.

\section{RESULTADOS E DISCUSSÃO}

Foi verificado efeito na produtividade da couve-flor em resposta às doses crescentes do Humoativo comercial (Figura 1a). A aplicação de 102 t.ha $^{-1}$ do produto proporcionou o maior rendimento entre as quantidades aplicadas, de 13 t.ha ${ }^{-1}$, inferior ao rendimento obtido com a aplicação de SFT que alcançou 16,6 t.ha ${ }^{-1}$. Na Figura 1b estão apresentados os dados de produtividade excluindo-se os dados da testemunha, evidenciando a resposta sobre a produtividade da couve-flor às doses de Humoativo.

As produtividades obtidas foram compatíveis com aquelas julgadas normais para a cultura, sendo que em solos com boas características, os rendimentos comerciais podem chegar a 18t/ha (SOUZA e RESENDE, 2003). Almeida et al (2007b) avaliando adaptação de cultivares de couve-flor em sistemas de produção orgânico obtiveram a maior produtividade com o híbrido Silver Streak, de 11,3 t.ha ${ }^{-1}$, seguida do cultivar White A e dos híbridos Yuki e Barcelona, com valores em torno de 8,4 t.ha ${ }^{-1}$. Em um trabalho para avaliar o efeito de doses de nitrogênio e boro sobre a incidência da podridão parda em couve-flor, Camargo et al.
(2008) obtiveram valores médios de 15,6 t.ha ${ }^{-1}$ com a cv. Julia, com respostas crescentes às doses de $\mathrm{N}$ e $\mathrm{B}$.

Figura 1 - Produtividade de couve-flor, cv. Júlia $\left(\mathrm{kg} \cdot \mathrm{ha}^{-1}\right)$, em função da aplicação de doses de Humoativo, considerando-se a testemunha (a) e sem testemunha (b) e com o produto referencial superfosfato triplo $(\mathrm{O})$
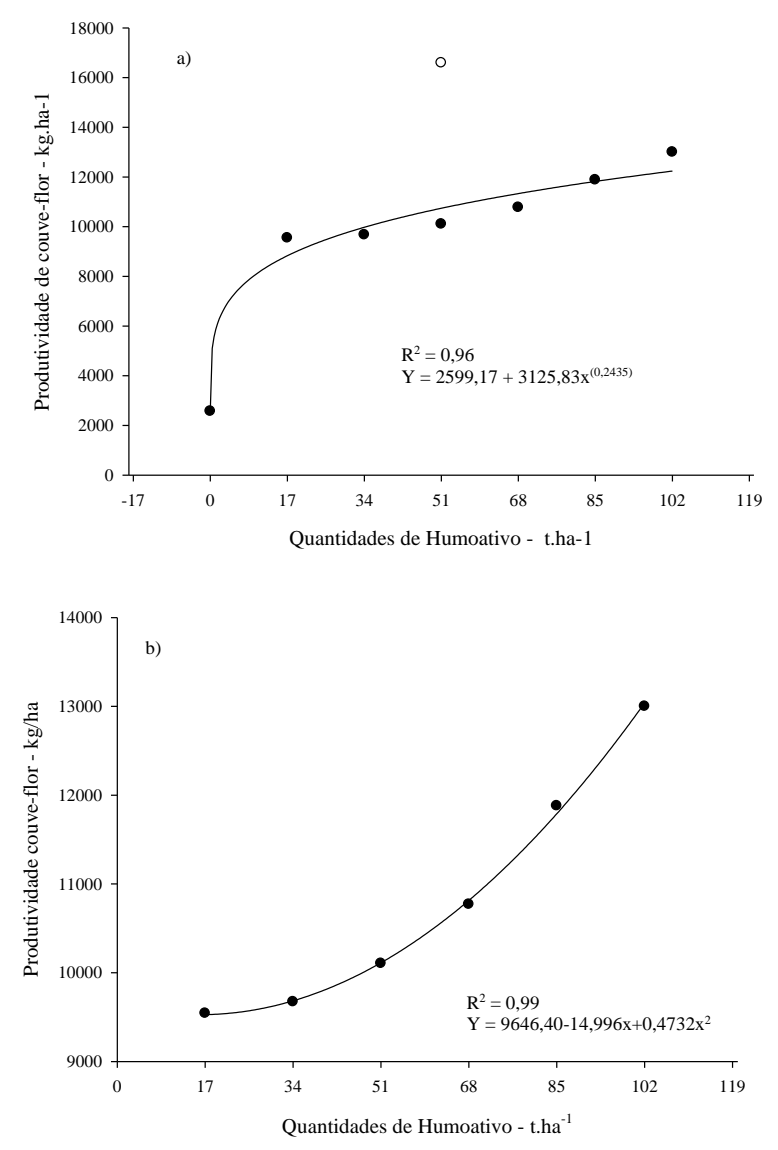

A produtividade obtida com a aplicação de fosfato solúvel na forma de SFT foi superior ao do tratamento com $51 \mathrm{t}^{-h a^{-1}}$, que continha a dose equivalente em $\mathrm{P}_{2} \mathrm{O}_{5}$. A dose de 102 t.ha ${ }^{-1}$ de Humoativo comercial, que equivalia em $\mathrm{P}_{2} \mathrm{O}_{5}$, ao dobro da recomendação, resultou na produtividade mais próxima àquela do tratamento superfosfato triplo. É provável que a localização do Humoativo, metade a lanço sobre a área total e metade na cova, tenha contribuído para os resultados observados. Na dose de 102 t.ha $^{-1}$, foram aplicados 51 t.ha ${ }^{-1}$ na cova, ficando esses, prontamente disponíveis para as raízes da couve-flor. É possível que muito pouco da parte aplicada a lanço tenha sido aproveitada pelas plantas, considerando o limitado crescimento das raízes da espécie. Godoy et al. (2012) testando doses de $\mathrm{K}_{2} \mathrm{O}$ em cobertura sobre a produção e qualidade de couve-flor cultivar Teresópolis Gigante observaram que não é necessária a aplicação deste nutriente em cobertura quando se faz a adubação de plantio e atinge-se o nível adequado deste nutriente.

De forma geral, não foram verificados empecilhos ao desenvolvimento das plantas (Figura $2 \mathrm{a}$ e $2 \mathrm{~b}$ ), indicando não ter havido limitação de nutrientes essenciais à cultura, mesmo na dose de 17 t.ha $^{-1}$, menor quantidade de Humoativo aplicada. 
Figura 2 - Vista geral do experimento (a) e característica produtiva da couve-flor (b) adubada com Humoativo
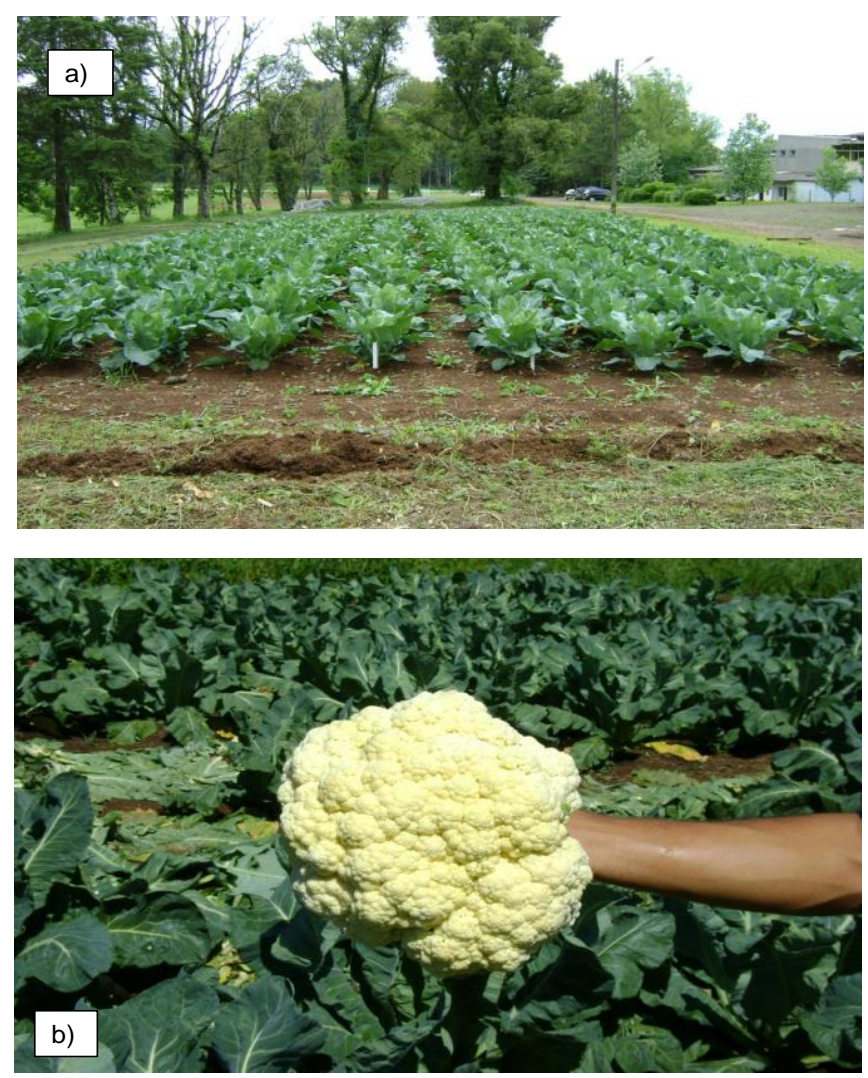

Houve efeito das doses de Humoativo sobre o teor de molibdênio no tecido vegetal da couve-flor, com redução na concentração de Mo nos tecidos foliares com a aplicação das doses (Figura 3). Possivelmente, este fato está relacionado ao efeito de diluição desse nutriente devido ao maior crescimento das plantas proporcionado pela aplicação do Humoativo, uma vez que na testemunha a produtividade foi baixa, ocorrendo uma maior concentração deste nutriente.

Figura 3 - Teores de Mo no tecido foliar da couve-flor após aplicação de diferentes doses de Humoativo

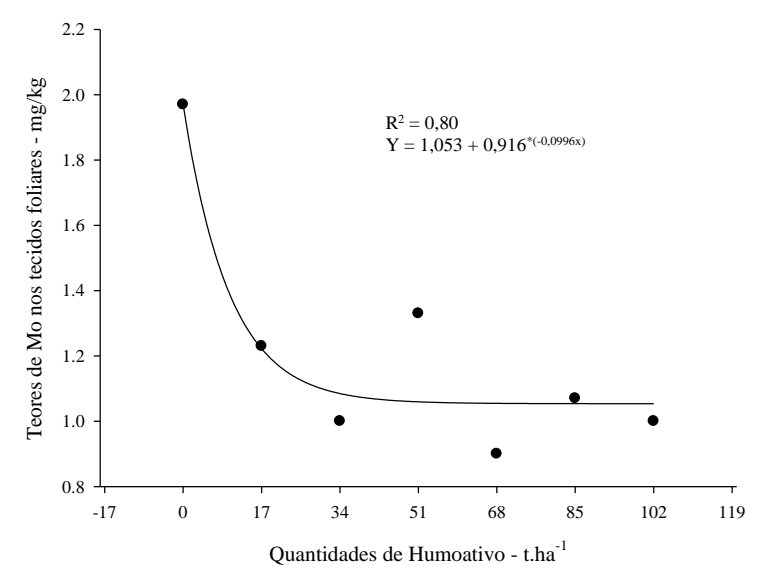

Pela natureza do Humoativo, de base orgânica, possuidor de alta concentração de radicais ionizáveis, o que lhe confere poder complexante (STEVENSON e ARDAKANI, 1972), uma possibilidade a considerar durante sua aplicação é sua característica de complexar alguns nutrientes, como o Mo, tornando-os dessa forma, menos disponível às plantas. Mordtvedt (2001) observa que há possibilidade de síntese de complexos orgânicos de metais a partir da reação de metais com subprodutos orgânicos da indústria de polpa de madeira e outros. A estabilidade da ligação quelato-metal determina, geralmente, a disponibilidade do nutriente aplicado para as plantas (ABREU et al., 2007). Para Mello e Minami (1999) de uma maneira geral, a calagem é suficiente para prevenir a deficiência deste micronutriente, entretanto em alguns casos, só é possível incrementar a produção com o fornecimento de sais de molibdênio. Diante da complexidade que se apresenta, é prudente o acompanhamento constante da cultura da couveflor com a aplicação do Humoativo, visando prevenir deficiências de Mo.

Na Tabela 3 estão apresentados os teores dos nutrientes no tecido das folhas da couve-flor, setenta dias após o transplante das mudas. Não houve efeito das doses para os teores de $\mathrm{N}, \mathrm{P}, \mathrm{K}, \mathrm{Ca}, \mathrm{Mg}$ e $\mathrm{S}$, nem tampouco para os teores dos micronutrientes avaliados $(\mathrm{Zn}, \mathrm{Cu}, \mathrm{Mn}, \mathrm{Fe})$, sendo que todos os valores se mantiveram próximos aos teores considerados adequados pela CQFS RS/SC (2004) para a cultura da couve-flor.

Houve efeito da aplicação de Humoativo apenas para o teor de sódio no tecido foliar da couve-flor, sendo o fenômeno foi mais bem representado por uma função polinomial do tipo quadrática (Tabela 3). Os níveis observados, mesmo que inicialmente crescentes, estão dentro de padrões normais para as folhosas (REUTER e ROBINSON, 1986).

Não foram observados efeitos das doses de Humoativo sobre os teores de metais pesados, no tecido foliar da couveflor em função dos diferentes tratamentos aplicados (Tabela 3). Os teores de $\mathrm{Cr}$ e $\mathrm{Hg}$ foram mais altos nas menores dosagens, incluindo a testemunha, e muito próximos daqueles observados com a aplicação do SFT, fertilizante comumente usado nos cultivos de couve-flor. Os teores de $\mathrm{Pb}$ e $\mathrm{Cd}$ observados ficaram abaixo daqueles detectáveis pelos instrumentos de avaliação, para todos os tratamentos. 
Tabela 3 - Teores de alguns macro e micronutrientes e de metais pesados nos tecidos das folhas de couve-flor cultivar Júlia, setenta dias após a germinação, em função de doses de Humoativo (HUM)

\begin{tabular}{|c|c|c|c|c|c|c|c|c|c|c|c|c|c|c|c|}
\hline HUM & $\mathrm{N}$ & $\mathrm{P}$ & $\mathrm{K}$ & $\mathrm{Ca}$ & $\mathrm{Mg}$ & S & $\mathrm{Zn}$ & $\mathrm{Cu}$ & $\mathrm{Mn}$ & $\mathrm{Fe}$ & $\mathrm{Cr}$ & $\mathrm{Na}^{1}$ & $\mathrm{~Pb}$ & $\mathrm{Cd}$ & $\mathrm{Hg}$ \\
\hline t.ha $^{-1}$ & \multicolumn{6}{|c|}{ g. $\mathrm{kg}^{-1}$} & \multicolumn{9}{|c|}{$\mathrm{mg} \cdot \mathrm{kg}^{-1}$} \\
\hline 0 & 30 & 4,4 & 24 & 5,6 & 1,8 & 6,8 & 34 & 5,0 & 13 & 59 & 2,5 & 118 & $<2$ & $<0,2$ & 0,023 \\
\hline 17 & 35 & 4,6 & 25 & 5,6 & 1,8 & 6,0 & 33 & 2,7 & 14 & 57 & 2,1 & 214 & $<2$ & $<0,2$ & 0,020 \\
\hline 34 & 37 & 5,0 & 25 & 5,1 & 1,9 & 7,1 & 39 & 3,0 & 15 & 66 & 1,3 & 230 & $<2$ & $<0,2$ & 0,027 \\
\hline 51 & 30 & 4,8 & 26 & 6,5 & 1,8 & 7,0 & 31 & 2,0 & 13 & 49 & 0,8 & 248 & $<2$ & $<0,2$ & 0,017 \\
\hline 68 & 26 & 4,2 & 26 & 4,9 & 1,7 & 6,5 & 28 & 2,0 & 12 & 46 & 1,4 & 239 & $<2$ & $<0,2$ & 0,013 \\
\hline 85 & 28 & 4,7 & 25 & 6,2 & 1,9 & 7,3 & 31 & 2,3 & 16 & 53 & 1,3 & 253 & $<2$ & $<0,2$ & 0,010 \\
\hline 102 & 26 & 5,7 & 28 & 7,1 & 1,9 & 7,6 & 32 & 3,3 & 14 & 48 & 3,3 & 246 & $<2$ & $<0,2$ & 0,013 \\
\hline Doses & ns & $\mathrm{ns}$ & $\mathrm{ns}$ & ns & ns & ns & $\mathrm{ns}$ & ns & ns & ns & $\mathrm{ns}$ & $\mathrm{Q}^{*}$ & - & - & - \\
\hline $\mathrm{R}^{2}$ & - & - & - & - & - & - & - & - & - & - & - & 0,86 & & & \\
\hline SFT* & 35 & 6,1 & 28 & 5,8 & 2,1 & 7,3 & 35 & 3,3 & 36 & 35 & 2,7 & - & $<2$ & $<0,2$ & 0,011 \\
\hline $\mathrm{CV} \%$ & 15,3 & 13,8 & 8,3 & 15,2 & 13,5 & 9,7 & 12,3 & 47,7 & 18,3 & 12,9 & 62,0 & 33,1 & - & - & 44,94 \\
\hline
\end{tabular}

ns = não significativo. Para doses, apresentada a curva de melhor ajuste $(\mathrm{L}=$ Linear simples ou $\mathrm{Q}=\mathrm{Quadrática})$ e $\mathrm{o}$ valor de $\mathrm{R}^{2} . *$ significância do teste $\mathrm{F}$ ao nível de 5\%; SFT- superfosfato triplo; $\mathrm{CV}$ - coeficiente de variação. ${ }^{1}$ Equação para $\mathrm{Na}=\mathrm{Y}=118,5952+4,0630 \mathrm{x}$ $-0,0295 x^{2}$

\section{CONCLUSÕES}

O uso do Humoativo comercial como fertilizante para a cultura da couve-flor, com a adição de boro nas covas, proporciona aumento da produtividade da cultura com o aumento das doses aplicadas;

Os teores dos metais $\mathrm{Cr}, \mathrm{Hg}, \mathrm{Pb}$ e $\mathrm{Cd}$ no tecido das folhas de couve-flor não são afetados pela aplicação de Humoativo TB® nas quantidades aplicadas nesse trabalho.

É possível obter-se produções normais de couve-flor por um ano sem aplicação adicional de Mo ao solo ou às plantas, em cultivos adubados com Humoativo.

\section{REFERÊNCIAS BIBLIOGRÁFICAS}

ABREU, C. A.; LOPES, A. S.; SANTOS, G. C. G Micronutrientes. In: NOVAIS, R. F.; ALVAREZ, V. H.; BARROS, N. F.; et al. (Eds). Fertilidade do solo. Viçosa: Sociedade Brasileira de Ciência do Solo, 2007. p.645-736.

ALMEIDA, H. C.; SILVEIRA, C. B.; ERNANI P. R. et al. Composição química de um resíduo alcalino da indústria de papel e celulose (dregs). Química Nova, São Paulo, v.30, n.7, p.1669-1672, 2007a.

ALMEIDA, K; CARVALHO, G.J.; GOMES, L.A.A. et al. Produção orgânica de couve-flor em sistema de plantio direto e convencional. Revista Brasileira de Agroecologia, Porto Alegre, v.2, n.1, p.1216-1219. 2007b.

CAMARGO, M.S.; MELlO, S.C.; FOLTRÁN, D.E.; CARMELLO, Q.A.C.. Produtividade e podridão parda em couve-flor de inverno influenciadas pelo nitrogênio e boro. Bragantia, São Paulo, v.67, n.2, p. 371-375, 2008.

CQFS - RS/SC. Manual de adubação e calagem para os Estados do Rio Grande do Sul e Santa Catarina. 10ed. Porto Alegre, RS: SBCS/Núcleo Regional Sul; Comissão de Química e Fertilidade do Solo, 2004. 400p.
GODOY, A. R.; SAlATA, A. C.; CARDOSO, A. I. I. et al. Produção e qualidade pós-colheita de couve-flor em função de doses de potássio em cobertura. Scientia Agraria Paranaensis, Cascavel, v. 11, n.2, p 33 - 42, 2012.

HOLZSCHUH, M. J.; BARTZ, H. R.; TREVISAN, J. N. et al. Parâmetros de rendimento de brassicaceas e a disponibilidade de fósforo em Planossolo Hidromórfico Distrófico arênico estimado pelo extrator de Mehlich-1. In: CONGRESSO BRASILEIRO DE CIÊNCIA DO SOLO, 29, Ribeirão Preto, 2003, Anais..;Ribeirão Preto, SBCS, 2003, CD-ROM.

JONES JUNIOR., J. B.; WOLF, B.; MILLS, H. A. Plant analysis handbook. Athens: Micro-Macro Publishing, 1991. $188 \mathrm{p}$.

KANO. C.; SAlATA, A. C.; HIGUTI, A. R. O. et al. Produção e qualidade de couve-flor cultivar Teresópolis Gigante em função de doses de nitrogênio. Horticultura Brasileira, Brasília, v.28, n.4, p. 453-457, 2010.

MELLO, S.C.; MINAMI, K. Efeitos do molibdênio e da calagem no crescimento da couve-flor cv. Shiromaru II. Piracicaba, Scientia agrícola, Piracicaba, v.56, n.1, p.235-237, 1999.

MOREIRA, D. A.; MARTINEZ, M. A.; SOUZA, J. A. R.; MATOS, A. T.; REIS, C. REIS, E. L. Determinação das características de resíduo sólido urbano aterrado. Engenharia Ambiental, Espírito Santo do Pinhal, v. 7, n. 1, p. 099-108, 2010.

MORTVEDT, J. J. Tecnologia e produção de fertilizantes com micronutrientes: presença de elementos tóxicos. In: FERREIRA, M.E.; CRUZ, M.C.; RAIJ, B, van; ABREU, C.A. (Ed.). Micronutrientes e elementos tóxicos na agricultura. Jaboticabal: CNPq/FAPESP/POTAFÓS, 2001. p. 237-251.

NELLESSEN, J. E.; FLETCHER, J. S. Assessment of published literature on the uptake, accumulation and 
translocation of heavy metals by vascular plants. Chemosphere, v. 27, n. 8, p. 1669-1680, 1993.

OLIVEIRA, F.C.; MATTIAZZO, M.E.; MARCIANO, C.R.; ABREU JÚNIOR, C.H. Fitodisponibilidade e teores de metais pesados em um Latossolo Amarelo distrófico e em plantas de cana-de-açúcar adubadas com composto de lixo urbano. Revista Brasileira de Ciência do Solo, Viçosa, v.26, n. 3; p 737-746, 2002.

PINTO FILHO, J.L.O.; SOUZA, M.J.J.B.; SANTOS, E.G.; GÊ, D.R.F.; CEZAR FILHO, P.Monitoramento dos teores totais e disponíveis de metais pesados no lixão do município de Apodi-RN. Revista Verde, Mossoró,v.7, n. 1, p. $141-147,2012$.

REUTER, D.J.; ROBINSON, J.B. Plant analysis: na interpretation manual. Inkata Press, Melbourne, Australia, 1986. p.218.

SILVA, C. A.; RANGEL, O.J.; DYNIA, J.F.; BETTIOL, W.; MANZATTO, C.V. Disponibilidade de metais pesados para milho cultivado em Latossolo sucessivamente tratado com lodos de esgoto. Revista Brasileira de Ciência do Solo, Viçosa, v.30, n.2, pp. 353-364, 2006.

SOUZA, J.L.; RESENDE, P. Manual de horticultura orgânica. Viçosa: Aprenda Fácil, 2003. 564p.

STEVENSON, F.J.; ARDAKANI, M.S. Organic matterreactions involving micronutrients in soils. In: MORTVEDT, J.J.; GIORDANO, P.M.; LINDSAY, W.L. (Eds.) Micronutrients in agriculture. Madison, Soil Science of America, p.79-114, 1972.

TEDESCO, M.J.; GIANELlO, C.; BISSANI, C.A. et al. Análise de solo, plantas e outros materiais. 2 ed. Departamento de Solos da UFRGS, 1995. 174p.

USEPA. Soil screening guidance: thecnical background document. Washington, EPA, Office of Solid Waste and Emergency Response, 1999. 